Journal club [L]

\section{A NOVEL GENETIC ARCHITECTURE OF} INFECTIOUS DISEASES

In the mid-1990s, the human genetics of infectious diseases was a field still in its infancy. In rare Mendelian conditions, the first causal mutations underlying classical primary immunodeficiencies (PIDs) were beginning to be discovered, but very few polymorphisms had been convincingly identified for common diseases thought to have a more complex predisposition. At the time, I was working on the human genetics of two common mycobacterial diseases, leprosy and tuberculosis, using classical genetic epidemiology approaches. Two companion papers published in 1996 by the groups of Mike Levin and Jean-Laurent Casanova greatly accelerated research in this field, and boosted my subsequent career.

These studies reported the discovery of interferon- $\gamma$ receptor 1 (IFNGR1) mutations in children with severe infections caused by weakly virulent mycobacteria, such as the bacille Calmette-Guérin (BCG) vaccine, a condition that was denoted as Mendelian susceptibility to mycobacterial diseases (MSMD). Both papers stressed the highly selective nature of this infectious predisposition, demonstrating a key role of IFN $\gamma$ in antimycobacterial defence in humans. These findings were at odds with studies of the corresponding knockout mice, which had increased susceptibility to many types of microorganism, which highlighted the importance of human studies in natural infection conditions. The selective susceptibility to mycobacteria (and Salmonella spp. in some cases) was confirmed following the identification of other MSMD-causing genes, all of which belonged to the IL-12-IFN $\gamma$ pathway.

These landmark papers led to a paradigm shift in the PID field, functioning as a starting point for the identification of new selective PIDs that increased susceptibility to invasive pneumococcal infections, herpes simplex encephalitis or infection with Candida spp. Last, but not least, these papers provided the foundation for the demonstration that tuberculosis is also a genetic disorder. Children with severe forms of tuberculosis were found to have mutations of genes already implicated in MSMD. I met Casanova after the publication of his IFNGR1 study and, together, we set up the Laboratory of Human Genetics of Infectious Diseases in which these studies were carried out. These papers were instrumental in persuading me, and the community working in this field, to consider a novel genetic architecture of infectious diseases.

Laurent Abel Imagine Institute, INSERM, Paris, France, and The Rockefeller University, New York,

USA. e-mail:Laurent.abel@inserm.fr The author declares no competing interests.

ORIGINAL ARTICLES Newport, M. J. et al. A mutation in the interferon-gamma-receptor gene and susceptibility to mycobacterial infection. N. Engl. J.Med. 335, 1941-1949 (1996) |Jouanguy, E. et al. Interferon-gamma-receptor deficiency in an infant with fatal bacille Calmette-Guerin infection. N. Engl.J. Med. 335, 1956-1961 (1996) 